\title{
Ein Verfahren zur Stabilitätsfrage bei Matrizen-Eigenwertproblemen
}

\section{Doctoral Thesis}

Author(s):

Schwarz, Hans Rudolf

Publication date:

1956

Permanent link:

https://doi.org/10.3929/ethz-a-000091928

Rights / license:

In Copyright - Non-Commercial Use Permitted 
Prom. Nr. 2603

\title{
Ein Verfahren zur Stabilitätsfrage bei Matrizen-Eigenwertproblemen
}

\author{
VON DER \\ EIDGENÖSSISCHEN TECHNISCHEN HOCHSCHULE \\ IN ZÜRICH \\ ZUR ERLANGUNG \\ DER WÜRDE EINES DOKTORS DER MATHEMATIK \\ GENEHMIGTE \\ PROMOTIONSARBEIT \\ VORGELEGT VON \\ HANS-RUDOLF SCHWARZ \\ dipl. Mathematiker ETH. \\ von $\mathrm{ZUURICH}$
}

Referent: Herr Prof. Dr. E. Stiefel

Korreferent: Herr Prof. Dr. H. Hopf

BASEL

Buchdruckerei Birkhäuser AG.

1956 


\title{
Ein Verfahren \\ zur Stabilitätsfrage bei Matrizen-Eigenwertproblemen
}

\author{
Von Hans-Rudolf Schwarz, Zürich'1)
}

\section{Einleitung und Problemstellung}

Zahlreiche technische Fragen führen auf das mathematische Problem, für das Matrizen-Eigenwertproblem $(A-\lambda E) x=0$ die Anzahl der Eigenwerte mit positivem Realteil zu bestimmen, wobei die Eigenwerte selbst meistens gar nicht interessieren. Diese Problemstellung nennt man kurz die Stabilitätsfrage. Um diese $\mathrm{zu}$ beantworten, sind verschiedene klassische Kriterien bekannt, welche aber alle vom charakteristischen Polynom $P(\lambda)$ ausgehen, also bedingen, dass dasselbe explizit ermittelt wird. So erwähne ich die Kriterien von RouTh $[1]^{2}$ ) und Hurwitz [2], welche in ihrer ursprünglichen Formulierung auf Polynome mit reellen Koeffizienten Anwendung finden, im übrigen aber gleichbedeutend sind. Das Ortskurvenkriterium von NYQuist [3] bestimmt auf graphischem Weg die Anzahl der Nullstellen der charakteristischen Gleichung $P(\lambda)=0$ mit negativem Realteil. Eine unmittelbare Folge davon ist das Lagen- oder Lükkenkriterium [4], welches aussagt, dass dann und nur dann sämtliche Nullstellen von $P(\lambda)=0$ negative Realteile aufweisen, wenn die Wurzeln von Realund Imaginärteil von $P(i y)=\operatorname{Re}(y)+i \operatorname{Im}(y)$ reell sind und sich gleichzeitig gegenseitig trennen. Das bekannteste numerische Verfahren zur Beantwortung der Stabilitätsfrage dürfte wohl die Methode der Sturmschen Kette [5] sein, wonach aus dem Real- und Imaginärteil von $P(i y)=\operatorname{Re}(y)+i \operatorname{Im}(y)$ durch fortgesetzte Division mit Rest eine Folge von Polynomen absteigenden Grades gebildet wird. Die Anzahl der Eigenwerte in der linken komplexen Halbebene $\operatorname{Re} z<0$ lässt sich dann aus dem Unterschied der Zeichenwechsel in der Polynomfolge für $y=-\infty$ und $y=+\infty$ angeben. Damit äquivalent ist das Stabilitätskriterium von WALL [6], welches die Aussage in die Form eines Kettenbruches kleidet. Daneben finden sich in der Literatur einerseits noch verschiedene numerische Verfahren, welche vermittels eines Reduktionsprozesses aus dem charakteristischen Polynom eine Folge von Polynomen bestimmen, aus denen die Frage beantwortet werden kann, und anderseits auch einige graphische Methoden.

1) Institut für angewandte Mathematik der ETH.

2) Die Ziffern in eckigen Klammern verweisen auf das Literaturverzeichnis, Seite 30. 
Auf der andern Seite führt jedes gangbare numerische Verfahren zur expliziten Entwicklung des charakteristischen Polynoms von $(A-\lambda E) x=0$ auf eine Folge von Polynomen, deren Grad sich bei jedem Schritt um 1 erhöht und die mit dem charakteristischen Polynom endigt. Ich verweise auf das Verfahren von Weber-Voetter [7], auf die Methode von Hessenberg [8] und den Biorthogonalisierungsprozess von LANczos [9], welche alle über eine Reihe von Polynomen aufsteigenden Grades zum charakteristischen Polynom führen.

Um dann die allein interessierende Stabilitätsfrage beantworten zu können, muss eine Polynomfolge absteigenden Grades ermittelt werden. Um den Weg über eine aufsteigende und dann über eine absteigende Kette von Polynomen zu vermeiden, in welcher das charakteristische Polynom als solches von höchstem Grad meist gar nicht interessiert, gab mir Herr Professor Dr. E. STIEFEL (ETH) die Anregung, folgende Frage zu untersuchen:

Problem: Man berechne das charakteristische Polynom $P(\lambda)$ aus der Matrix $A$ über eine solche aufsteigende Kette von Polynomen, aus welcher schon selbst die Stabilitätsfrage beantwortet werden kann.

Dieser Wunsch führte zu einem Verfahren, unter Umgehung des charakteristischen Polynoms die gegebene Matrix $A$ mit Hilfe von elementaren Transformationen auf eine Normalform zu bringen, deren Elemente allein schon das Stabilitätsproblem lösen. Die beschriebene Methode weist den Vorteil auf, dass die anzuwendenden Rechenoperationen von grosser Einheitlichkeit sind, was das Programmieren auf Rechenautomaten erleichtert. Bei der klassischen Methode der Sturmschen Kette stützt sich die ganze Entscheidung der Stabilität auf eine längere Rechnung, welche vom charakteristischen Polynom ausgeht. Die Koeffizienten dieses Polynoms enthalten sicher weniger Information als die ursprünglich gegebenen Matrixelemente. Es ist zu hoffen, dass unsere Methode, die die Matrixelemente in mehr direkter Weise verwendet, in manchen Fällen numerisch stabiler verläuft. $\mathrm{Ob}$ diese Hoffnung berechtigt ist, können allerdings erst umfangreichere Versuche entscheiden.

An dieser Stelle möchte ich den Herren Professoren Dr. E. STIEFel und Dr. H. Rutishauser, die mit ihren wertvollen Hinweisen zum Gelingen dieser Arbeit beigetragen haben, meinen besten Dank aussprechen.

\section{Das Stabilitätskriterium}

\section{Das Kettenbruchkriterium von WaLL [6]}

Es sei $P(z)=z^{n}+c_{n-1} z^{n-1}+c_{n-2} z^{n-2}+\cdots+c_{1} z+c_{0}$ ein Polynom $n$-ten Grades mit komplexen Koeffizienten $c_{k}=p_{k}+i q_{k}$. Aus diesem bilde man $Q(z)=\left[P(z)-(-1)^{n} P(-z)\right] / 2$, worin $P(z)$ das Polynom mit den konjugiert 